\title{
Statistical Control of Accuracy at Machining
}

\author{
Angel Lengerov ${ }^{1}$, Galina Nikolcheva², Lyubomir Lazov ${ }^{3}$ \\ ${ }^{1}$ Department of Machine Tools and Mechanical Engineering, Technical University, Plovdiv, Bulgaria, \\ ${ }^{2}$ Department of Machine Tools and Mechanical Engineering, Technical University, Sofia, Bulgaria, ${ }^{3}$ Rezekne \\ Academy of Technologies, Latvia
}

\begin{abstract}
This research examines the statistical method for quality control. The accuracy of parts is controlled statistical evaluation - an average and a dispersion of the indicator for accuracy. This paper reports a method for quality control of parts of average indicators and algorithm for quality control of the average and dispersion of samples of small size. It proposes a criterion of applicability of statistical adjustment of accuracy by sampling for control of the average data of the quality indicator. It proves the influence of the dispersion of the quality indicator for impairing the quality of the process.
\end{abstract}

Keywords: dispersion, Statistical Process Control, quality indicator, sample.

\section{INTRODUCTION}

In today's computer-aided manufacturing environment, one of the main objectives of quality control is to achieve higher accuracy of machined workpieces. One way to improve the workpiece accuracy is to utilize concept of deterministic metrology by focusing on the machine tool and the cutting process to control the cutting tool position accurately in the workspace of the machine tool. Another way to improve accuracy in the production of machines parts is to use methods of statistical process control. These methods allow to apply in process control systems. By implementing statistical process control (SPC), which involves the gathering and analysis of data to determine which machining process is most suitable for a workpiece, we can ultimately improve quality and reliability, and reduce operating costs.

SPC is a commonly used control method of manufacturing processes which is effective in controlling the fluctuations of the manufacturing process. Statistical Process Control (SPC) techniques, when applied to measurement data, can be used to highlight areas that would benefit from further investigation. These techniques enable the user to identify variation within their process. SPC utilizes statistical methods to monitor manufacturing processes with an aim to maintain and improve the product quality while decreasing the variance. Understanding this variation is the first step towards quality improvement. SPC techniques are tools for highlighting this unusual behavior. Automated data collection, low cost calculation, and demands for higher quality, lower cost, and greater reliability are the cause of using SPC [4].
Past 30 years, many researchers as Georgiev and Kuzmanov [3], Woodall and Montgomery [9], and Stoumbos et al. [7] develop and implement schemes for statistical control of production quality improvement and productivity. SPC helps define the capability of the stable process to judge whether it is operating at an acceptable $[2,5]$.

This research examines the statistical method for quality control. The aim of this article is to create a method and algorithm to control the quality of parts by the average data of indicators and dispersion of samples of small size. The machined parts accuracy affects to quality of the machine tools. The two terms often used with quality are error and accuracy. Error represents deviations of the cutting tool point from the theoretical position on the part in order to machine part dimensions to specified tolerance. Accuracy is defined as the part's conformance to the specifications in the manufacturing drawing or CAD MODEL [8]

Section 2 describes statistical control methods of the accuracy of machining, section 3 describes the developed methodology and algorithm for control accuracy in the manufacture of machine parts by controlling the average data and by dispersion of samples of small size.

\section{METHODS FOR STATISTICAL CONTROL}

There are various sources of errors, which result in inaccurate details. Main indicators of the quality of the parts are geometric shape, dimensional accuracy, relative position of their surfaces and material properties. These indicators are measurable physical quantities. For each of them a nominal size and the size of the deviation are referred. These quality http://dx.doi.org/ 10.17770/etr2017vol3.2596 
characteristics are controlled by statistical evaluation of the processing of the series of identical parts. The control is carried out with different sample size small and big samples [4]. The method of small samples applies to processes that are scheduled rarer setups. The information necessary for the analysis need to be collected in a long time [3,7]. Samples are up to 10 and they are taken at regular intervals of 30 to 60 minutes.

The method of the big sample is applied to processes with high intensity of the dominant systemic factors. The method of the big sample is applied to processes with high intensity of the dominant systemic factors. They require more frequent setups or presetting of the technological system. Controls all parts in the sequence of their execution [3,7]. The sample size is 50 to 200 .

At small samples - to control the nominal value using the evaluation on the average of the controlled indicator, at big samples - the regression equation for the average data.

\section{Reasons for deviation of the quality}

The types of deviation of the quality are shown graphically in Fig. 1. In the case of Fig. $1 a$, as a result of the systematic increase in the average $\bar{A}$ the field of scattering has reached the upper limit of the tolerance zone. If the process continued after time $\tau_{k p}$ there is probability to produce poor quality parts. In the case of Fig. $1 b$, with continued work increased the average $\bar{A}$ and the dispersion. Both factors together lead to problems in the process.

In the case of Fig. 1c, the initial setting process is of poor quality. It reduces operating time until the next presetting. The purpose is through the control to establish the moment that should be stopped setup process.

Where production has a big series, with accumulated statistical data on the process and the process is stable then the control can be limited to statistical evaluation, which reflects the change of the center of grouping of quality indicator as a function of time. The dispersion is a constant and with a certain size.

When there is no a priori statistical data about the characteristics of the process, then is required at realtime to identify the regression equation and the probable field of scattering. Parallel is implemented and specific algorithm for process control. This approach applies to the frequent change of the manufactured products and relatively small batches.

For the control of process is necessary to determine the criterion by which to evaluate the probability of deviations of the quality. The diagrams in Fig. 1 show that this is the time, that this criterion is the time $\tau_{k p}$ at which the field a scattering of the indicator for quality passes limit of the tolerance zone. The problem is that the field a scattering is determined by the theoretical law distribution and the dispersion of quality indicator as a random variable. For a normal distribution is accepted the limits of scattering to be $\omega= \pm 3 \sigma=6 \sigma$. The dispersion $D(x)$ and the standard deviation $\sigma$ are characteristics of the overall population - the number of parts $n \rightarrow \infty$. In practice, the process is monitored with limited sample size of the overall population. Therefore as a criterion not used the limits of the tolerance zone and control limits within the tolerance zone. The position of these limits are determined by what statistical evaluations of mathematical expectation $M(x)$ and the dispersion $D(x)$ are used for ongoing quality control.

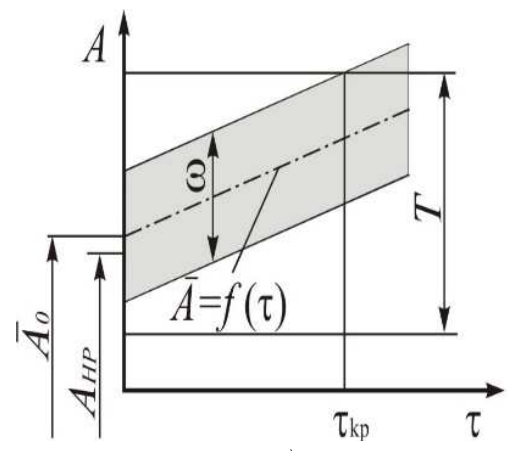

a)

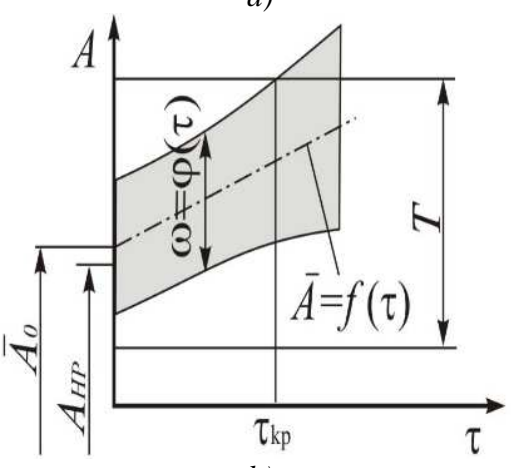

b)

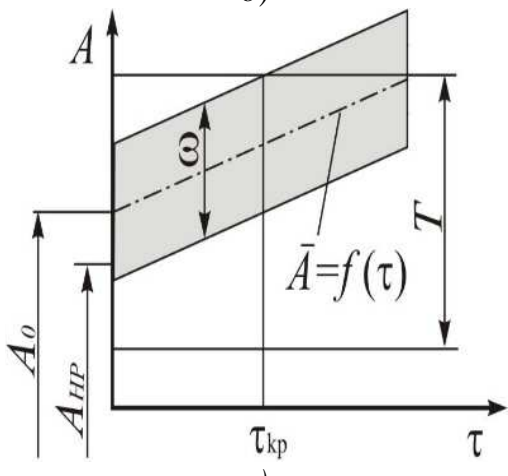

c)

Fig. 1. Reasons for the deviation of quality

\section{Control according to the average indicator for quality}

The change in the average data is caused under the influence of factors such as tool wear, thermal deformation, changing the cutting conditions and setting the technological system. Probability of 
obtaining a reject occurs when average value approached the limits of the tolerance zone. The limit positions of the average $\bar{A}$ of the sample are shown in Fig. 2, where the distribution of the overall population is indicated by the dotted line, and the sample - a continuous line.

As statistical evaluation, the average $\bar{A}$ scattering around the mathematical expectation $M$ of the overall population within the limits is

$$
\bar{A}=M \pm \frac{3 \sigma}{\sqrt{n}}
$$

where $\sigma$ is the standard deviation of overall population; $\mathrm{n}$ - the number of parts in a sample. The smallest permissible value of $\bar{A}$ is shown in Fig. $2 a$. Then the overall population in probability may be located on the border of reject, taking into account measuring error $\omega_{и з м}$ at control. Probability of reject is obtained when:

$$
\bar{A}<A+E I+\omega_{\text {изм }}+\frac{\omega}{2}+\frac{3 \sigma}{\sqrt{n}}
$$

Therefore, the lower control limit $K_{\min }$ is

$$
\begin{aligned}
& K_{\text {min }}=A+E I+\omega_{u з 3}+\frac{\omega}{2}+\frac{3 \sigma}{\sqrt{n}}= \\
& =A+E I+\omega_{u з 3}+3 \sigma\left(1+\frac{1}{\sqrt{n}}\right)
\end{aligned}
$$

Similarly of Fig. $2 b$ is defined upper control limit $K_{\max }$

$$
K_{\text {max }}=A+E S-\omega_{\text {изм }}-3 \sigma\left(1+\frac{1}{\sqrt{n}}\right)
$$

When the average $\bar{A}$ reaches one of the control limits with tend to pass, then having to make setup. The condition for stopping the process to make a setup is:

$$
K_{\min } \geq \bar{A} \geq K_{\max }
$$

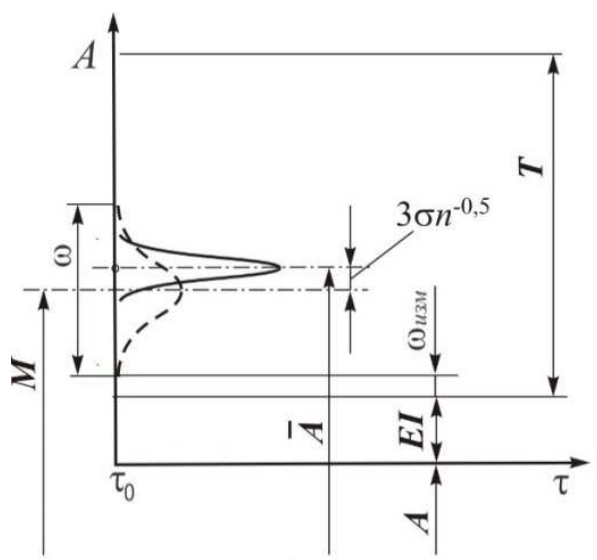

a)

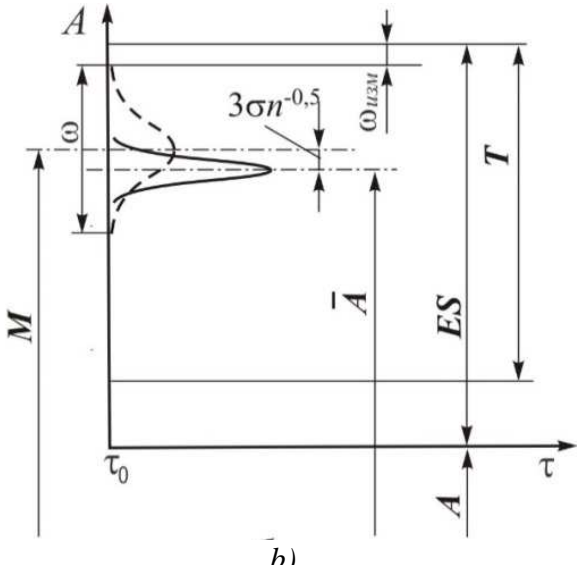

b)

Fig. 2. Limit positions of the scattering on the overall population

From (2) and (3) that to determine the control limits is necessary to know the dispersion of the overall population. For this purpose, it is the statistical evaluation of the dispersion $S$. The relationship between $S$ and the standard deviation $\sigma$ is the formula for the probable field scattering of the overall population:

$$
\omega=2 s k_{\gamma, P, n}=6 \sigma
$$

Therefore:

$$
\sigma=\frac{k_{\gamma, P, n}}{3} s
$$

The bigger is the number of parts of which is determined $S$, the closer will be up to standard deviation $\sigma$. It follows that at statistical control with small current sampling to determine the dispersion of the overall population do not use the average dispersion sample. Each sample dispersions is determined by a small number of parts and unjustified will underreport the probable field of the overall population, and hence will narrow and the zone between the control limits. This creates abnormal conditions of control process, and in certain cases impossible. Therefore the dispersion should be determined from all measured products during the observation. For example, if it is 10 samples, each of which has 5 products, the dispersion will be determined by a total of 50 articles instead of 5 . The evaluation of the dispersion of statistical data for small current sample equals to: 


$$
\begin{gathered}
s^{2}=\frac{\sum_{j=1}^{N_{j}} \sum_{i=1}^{n_{j}}\left(A_{j, i}-\bar{A}_{j}\right)^{2}}{\left(\sum_{j=1}^{N_{j}} n_{j}\right)-1}= \\
=\frac{\sum_{j=1}^{N_{j}} s_{j}^{2}\left(n_{j}-1\right)}{\left(\sum_{j=1}^{N_{j}} n_{j}\right)-1}
\end{gathered}
$$

where: $N_{j}$ is the number of samples, $n_{j}$ is the size of the sample with a number $\mathrm{j}, s_{j}^{2}$ is the sampling dispersion with number $j$.

$$
s^{2}=\frac{n-1}{n N_{j}-1} \sum_{j=1}^{N_{j}} s_{j}^{2}
$$

The range, which varies the average of the samples so that the process to ensures the quality equals to

$$
\begin{aligned}
& \Delta \bar{A}=K_{\text {max }}-K_{\text {min }}= \\
& =T-2 \omega_{\text {изм }}-6 \sigma\left(1-\frac{1}{\sqrt{n}}\right),
\end{aligned}
$$

where $T-2 \omega_{\text {изм }}=T_{\text {mex }}$ is called tolerance for machining and is accepted to be $0,8 T$. To be able to controlled the process is necessary $\Delta \bar{A}>0$.

Then from equation 8 is obtained condition:

$$
\sigma<\frac{0,8 T}{6\left(1+\frac{1}{\sqrt{n}}\right)} ; \quad \omega<\frac{0,8 T}{1+\frac{1}{\sqrt{n}}}
$$

For example, If $n=5$, then are calculated $\omega<0,55 T$. At $\omega=0,4 T$, the range of adjustment is calculated $\Delta \bar{A}=0,22 T$, which is a relatively narrow range when the process has a dominant systematic factor.

\section{Control algorithm on average $\bar{A}$}

The algorithm is given below.

- Step 1. Input data: part, operation, surface, dimensions A, T, EM, the method of small samples, size of the samples, time between samples $\tau$, process characteristics $\sigma, \omega, \omega_{\text {изм. }}$

- Step 2. Verification of applicability of the method: inequality (9). If satisfying, the algorithm continues. Otherwise go to step 1 and change an input.

- Step 3. Calculation of the control limits by the formulas (2), (3).

- Step 4. Input number of first sample $j$.
- Step 5. Entering the dimensions $n_{j, i}$ parts of the sample.

- Step 6. Calculation of $\bar{A}_{j}, s_{j}^{2}, s$.

- Step 7. If $K_{\text {min }} \geq \bar{A} \geq K_{\text {max }}$, then the process stops. Otherwise, the algorithm continues.

- Step 8. Input number of next sample $j=j+1$.

- Step 9. Repeat steps 2 to 8 to satisfy the inequality (4), wherein the process is interrupted for setup.

Is another new cycle for many small current samples.

It is possible that the algorithm can be extended to determine the regression equation and the dispersion of the set of processed samples. This information can be displayed graphically and be entered into the database for use in future developments.

\section{Control by dispersion of the indicator for quality}

In cases where the dispersion is a key indicator of the quality of production, it is used to control the quality process. Most often the control on dispersion is combined with the control on average data. In this variant both indicators have a shared role in the quality of products. Both methods of control are based on a sample control for the dispersion of quality indicator.

The main purpose of monitoring is to establish whether the dispersion is statistically constant, whether occurs systemic change or is there an abrupt change of dispersion.

The check for the constancy of the dispersion is limited to verifying the hypothesis of equality of the dispersions. For samples of the same size are applied the criteria of Cochrane [1] wherein is verified the equality of the series of dispersions, when the samples have different size are applied the criteria of Bartlett [3].

During the process, the inspection begins after the third sample and is done after each subsequent sample. For the application of this method computing resources for a quick statistical analysis, such as modern computer systems are needed.

At the moment at which the null hypothesis $H_{0}: s_{1}^{2}=s_{2}^{2}=s_{3}^{2}=\ldots=s_{m}^{2}=s^{2}$ is not confirmed, this is a signal for an abrupt or for systemic change of the dispersion. If not passed limits for a quality produce, then are checked another 2-3 samples in short time. After checking on homogeneity, the sample with significantly different values of dispersion is excluded from the analysis. If the null hypothesis is confirmed, it implies that the abrupt change of dispersion is momentary phenomena and the process can continue (do not lose accuracy). If the next samples don't confirm the null hypothesis, it is signals for deviation accuracy of the process. 
At processes where control is performed only by dispersion of the quality indicator, then the dispersion of all samples is determined by (6) or (7). The obtained dispersion is compared with the permissible for the process in order to evaluate the need to setup. When the dispersion is used together with the average indicator of quality, should be monitored change of $S$ by (6) and respectively of $\sigma$. With the new values after each sample are recalculated the control limits $\left(K_{\text {max }}, K_{\text {min }}\right)$. With the increase in the dispersion is narrowed the range between the control limits and is increased the probability the average $\bar{A}$ of the qualitative indicator of the sample reach the limits. Thus, the change of the dispersion reflects the moment when the process is interrupted for a setup or a new setting.

\section{CONCLUSIONS}

Statistical Process Control (SPC) techniques enable the user to identify variation within their process. The accuracy of parts is controlled statistical evaluation - an average and a dispersion of the indicator for accuracy. Method for quality control of parts by average indicators is created. A criterion of applicability of statistical adjustment of accuracy by sampling for control of the average $\bar{A}$ of the quality indicator is proposed. The influence of the dispersion of the quality indicator for impairing the quality of the process is proven.
The proposed algorithm for control accuracy in the manufacture of machine parts is based of the average data and dispersion of samples of small size. It takes into account the influence of the dispersion during the process. Using SPC technique helps us to easily understand when the permanent improvement occurs in the processes.

\section{REFERENCES}

[1] Cochran W. Sampling Techniques. Third ed. Wiley, 1977.

[2] Danov T. Statistical methods for quality control. Technika. Sofia.Bulgaria. 1987.

[3] Georgiev B, Kuzmanov T. Statistical methods for analysis and quality of technological processes in mechanical engineering. EXP-PRES. Gabrovo. Bulgaria. 2006.

[4] Fazeli AR, Sharifi E. Statistical Control and Investigation of Capability of Process and Machine in Wire Cut EDM Process of Gas Turbine Blade Airfoil Tip. Engineering. 2011; 3: 260265. http://www.SciRP.org/journal/eng)

[5] Montgomery DC. Introduction to Statistical Quality. John Wiliy\&Sons. Hoboken.1996.

[6] Rachev R, Lengerov A, Levicharov G. Providing manufacturability of engineering products. Plovdiv. Bulgaria.2016.

[7] Stoumbos ZG, Reynolds MR, Ryan TP and Woodall WH,. The State of Statistical Process Control as We Proceed into the 21st Century," Journal of the American Statistical Association.2000; Vol. 95. No. 451: 992-998.

[8] Stephenson DA, Agapiou JS. Metal Cutting Theory and Practice. Third ed. Marcel Dekker.2016.

[9] Woodall WH, Montgomery DC. Research Issues and Ideas in Statistical Process Control, Journal of Quality Technology.1999; Vol. 31. No. 4: 376-386. 